\title{
Rotational resonance of magnetoinductive waves: Basic concept and application to nuclear magnetic resonance
}

\author{
L. Solymar ${ }^{\mathrm{a})}$ \\ Department of Electrical and Electronic Engineering, Imperial College of Science, Technology \\ and Medicine, Exhibition Road, London SW7 2BT, United Kingdom \\ O. Zhuromskyy, O. Sydoruk, and E. Shamonina \\ Department of Physics, University of Osnabrück, D-49069 Osnabrück, Germany \\ I. R. Young and R. R. A. Syms \\ Department of Electrical and Electronic Engineering, Imperial College of Science, Technology \\ and Medicine, Exhibition Road, London SW7 2BT, United Kingdom
}

(Received 16 August 2005; accepted 4 May 2006; published online 27 June 2006)

\begin{abstract}
Magnetoinductive waves propagating along a set of resonant metamaterial elements are studied under the condition when the wave travels round a closed circular path and the total phase shift is an integral multiple of $2 \pi$. The resonant frequency of the circulating wave is shown to be related to the resonant frequency of the element via the known dispersion relationship. The currents in the elements are determined with the aid of the impedance matrix when the excitation is by a rotating magnetic dipole located at the center of the structure. It is shown that the power taken out from one element in the loop may approach $N$ times that from a single element, where $N$ is the number of elements, provided the quality factor of the individual elements is sufficiently high and suitable modifications are made to nearby elements. Potential applications to magnetic resonance spectroscopy are discussed. (C) 2006 American Institute of Physics. [DOI: 10.1063/1.2209031]
\end{abstract}

\section{INTRODUCTION}

Metamaterials research seeks to understand how the electromagnetic properties of a material can be changed, by inserting a set of usually identical elements in a random or periodic manner. The topic was popular after the second World War for applications in radar antennas and was further studied in the 1980s and 1990s in the search for chiral materials. A recent stimulus was the reevaluation of three seminal papers on, respectively, negative refractive index material by Veselago, ${ }^{1}$ the split ring resonator ${ }^{2}$ (also known as slotted tube resonators ${ }^{3}$ and loop-gap resonators ${ }^{4}$ ), and metallic rod structures ${ }^{5}$ which offer low plasma frequencies. In 1999 Pendry et $a l^{6}{ }^{6}$ showed that split ring resonators (SRRs) may produce negative permeability within a certain frequency range near to their resonant frequency. Smith et al. ${ }^{7}$ then combined SRRs and rods to produce a material with both negative permittivity and permeability. Later they validated Veselago's prediction of negative refraction. ${ }^{8}$ Though some reservations have been expressed, negative refraction essentially follows from the existence of backward waves, which have formed the basis of a number of microwave devices. ${ }^{9}$ In fact, Lindell et al. ${ }^{10}$ have suggested that negative index media should be called backward wave media.

Theoretical approaches to metamaterials often use an effective medium approximation, which relies on the averaging of microscopic fields. ${ }^{6,11}$ The theory has been able to predict the reflection and transmission of a transverse electromagnetic wave incident on a slab of metamaterial and

\footnotetext{
${ }^{a)}$ Author to whom correspondence should be addressed; electronic mail: laszlo.solymar@eng.ox.ac.uk
}

measured results have been reasonably close to the predicted ones. However, there have been no detailed comparisons between theory and experiment concerning the region of validity of the effective medium approximation, e.g., how small or how far from each other the elements should be, and how large the overall size of the medium should be. A limitation of effective medium theory was soon apparent in that it could not account for the presence of other than transverse electromagnetic (EM) waves.

Such additional waves may be excited by incident EM waves (or sometimes just by voltage sources), but they can have an entirely separate existence. They may propagate along a set of metallic nanoparticles, ${ }^{12-14}$ loaded electric dipoles, ${ }^{15}$ or capacitively loaded loops. Due to the magnetic coupling between the elements, the waves propagating along the latter structure have been termed magnetoinductive (MI) waves. ${ }^{16}$ The simplest explanation of their properties is in terms of nearest neighbor interactions analogous to the classical description of acoustic waves in crystals. ${ }^{17}$ A more detailed analysis of MI waves ${ }^{18}$ was followed by experiments on capacitively loaded loops and Swiss rolls. ${ }^{19,20}$

Applications of MI waves have been reported for delay lines, ${ }^{21}$ phase shifters, ${ }^{22}$ and microwave lenses. ${ }^{23}$ An early application was for signal guidance in magnetic resonance imaging (MRI), to try and improve the signal to noise ratio. ${ }^{24}$ As will be discussed later, the relevance of the work described here to MRI is currently unproved but there are forms of magnetic resonance spectroscopy (MRS) in vivo for which it may have relevance. Arrays of coils have long been used for MR signal detection. However, it was generally argued that interaction between the elements should be eliminated. ${ }^{25}$ For nearest neighbors, this was achieved by 


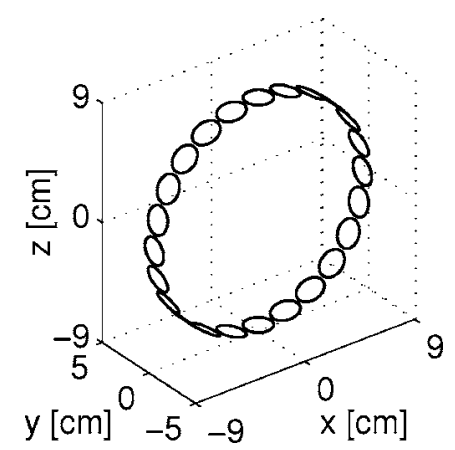

FIG. 1. Rotational resonator composed of 24 capacitively loaded loops.

overlapping adjacent loops. Other MR detector designs do rely on magnetic and electric interactions and are realized by a combination of lumped and distributed elements. Examples are the low- and high-pass birdcages ${ }^{26}$ and the dome resonator, ${ }^{27}$ used for imaging the human head.

It should be noted that in each case the physical basis of dipole waves, MI waves, and the waves exploited in MRI detectors is the interaction between resonant circuits, and was first analyzed many years ago. For example, Nefedov and Tretyakov ${ }^{22}$ recently found that Silin and Sazonov ${ }^{28}$ derived the dispersion equation for waves on magnetically coupled resonant circuits in their book on slow-wave structures published in Russia as early as 1966. The same dispersion equation also appears in an earlier book. ${ }^{29}$

This paper has been inspired by several disparate sources. Two have already been mentioned, the existence of waves on magnetically coupled elements and the need to improve MR detection. A third is a recent investigation of coupling between electromagnetic and MI waves, ${ }^{30}$ and a fourth is the ring resonator, which operates on the principle that the total phase shift that a wave accumulates round a closed path should be an integral multiple of $2 \pi$. An example-the circular strip-line resonator ${ }^{31}$-is well known in microwaves. An important fifth source is the cavity magnetron of Randall and Boot, ${ }^{32}$ the high power microwave source that did so much for World War II radar, and which is operated by coupling power from a circulating electron beam to an electromagnetic wave propagating round a ring of coupled cavity resonators.

Combining these ideas, we propose here a ring resonator in which MI waves travel round a circular structure consisting of magnetically coupled, capacitively loaded loops. The frequency range is not significant, as long as all the dimensions are small relative to the electromagnetic wavelength. Nonetheless, we have in mind the tens of megahertz region of MRI. The geometry is shown in Fig. 1; it has analogies to phased arrays in MRI, but the principle is actually quite different. Here, we make maximum use of the mutual inductances to achieve a traveling MI wave resonance, which we shall call "rotational resonance." We couple the resonant MI wave to a centrally located, rotating magnetic field and then show how, using the magnetron principle, a power far in excess of that available from an isolated element may be delivered from a suitably modified element of the ring. This development promises possible advances in MR signal detection. In Sec. II we introduce the basic properties of MI

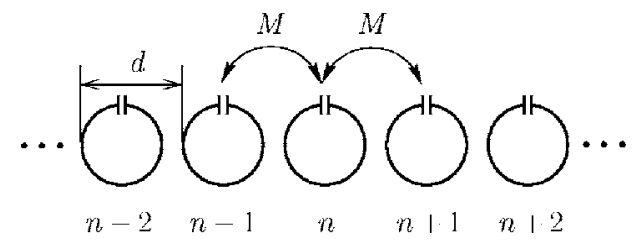

FIG. 2. Schematic representation of nearest neighbor coupling between loops.

waves and derive the conditions for rotational resonance. Detailed analysis and numerical results are presented in Sec. III. Possible MR applications are discussed in Sec. IV and conclusions are drawn in Sec. V.

\section{BASIC PROPERTIES OF MAGNETOINDUCTIVE WAVES}

Magnetoinductive waves are known to exhibit a variety of dispersion characteristics which may be anisotropic and may represent both forward and backward waves. ${ }^{18,19}$ They propagate along a set of magnetically coupled elements, which we shall take in the form of capacitively loaded metallic loops. In the simplest one-dimensional case, and in the approximation that only the nearest neighbor interactions are important, the relation between the currents in neighboring elements may be obtained by applying Kirchhoff's voltage law to the $n$th loop (see Fig. 2) as

$$
Z_{0} I_{n}+Z_{m}\left(I_{n+1}+I_{n-1}\right)=0 \text {. }
$$

Here $Z_{0}=R+j(\omega L-1 / \omega C)$ is the self-impedance of the loop, $L, C$, and $R$ are its inductance, capacitance, and resistance, $\omega$ is the angular frequency, and $Z_{m}=j \omega M$ is the mutual impedance between nearest neighbors, where $M$ is the mutual inductance. $I_{n}$ is the current in the $n$th loop. Assuming a traveling wave in the form $I_{n}=I_{0} \exp (-j n k d)$, where $d$ is the distance between the elements, Eq. (1) modifies to

$$
\cos (k d)=-Z_{0} / 2 Z_{m} \text {. }
$$

Since the right-hand side of the above equation is complex, $k$ must also be complex, so that $k=\beta-j \alpha$, where $\beta$ and $\alpha$ are the propagation and attenuation coefficients. Equation (2) reduces then to two real equations which give the frequency dependence of $\beta$ and $\alpha$

$$
\begin{aligned}
& \cos (\beta d) \cosh (\alpha d)=\kappa^{-1}\left[\left(\omega_{0}^{2} / \omega^{2}\right)-1\right], \\
& \sin (\beta d) \sinh (\alpha d)=\left(\kappa Q_{0}\right)^{-1} .
\end{aligned}
$$

Here $\kappa=2 M / L$ is the coupling coefficient, $\omega_{0}=(L C)^{-1 / 2}$ is the resonant frequency of the loop and $Q_{0}$ is its quality factor. For low attenuation, the term $\cosh (\alpha d)$ may be ignored in Eq. (3) and $\alpha d$ may then be found from Eq. (4) as

$$
\alpha d=\left[Q_{0} \kappa \sin (\beta d)\right]^{-1} \text {. }
$$

Next, we shall look at the arrangement shown in Fig. 1 where $N$ coupled loops lie on the perimeter of a circle of radius $r$. If $N$ is large enough, the distance between the elements is related to $r$ by $d=2 \pi r / N$. A wave propagating around a circular structure has a resonance when the circumference of the circle is equal to an integral multiple of the 
wavelength. This occurs when $2 \pi r=s \lambda$ where $s$ is an integer and $\lambda=2 \pi / \beta$ is the wavelength of the MI wave. Hence $\beta d$ $=2 \pi s / N$, and the frequency of the rotational resonance, from Eq. (2), is

$$
\omega_{r} / \omega_{0}=[1+\kappa \cos (2 \pi s / N)]^{-1 / 2} .
$$

We shall briefly look at higher order resonances in Sec. III E. Until then, we shall assume $s=1$. It may be seen from Eq. (6) that the relative value of the fundamental resonance depends only on $\kappa$ and $N$. For the geometrical arrangement of Fig. $1, \kappa$ is negative. In the numerical examples to follow later we shall take $N=24$ and $r=85 \mathrm{~mm}$, yielding $\kappa=-0.11$. From Eq. (6) we then find that $\omega_{r} / \omega_{0}=1.058$, and full numerical calculations yield $\omega_{r} / \omega_{0}=1.057$.

\section{ANALYSIS}

\section{A. Excitation of the rotational resonance}

We now assume that the circular arrangement of resonant loops is excited by a nucleus under magnetic resonance conditions. This case can be modeled by a magnetic dipole rotating at an angular velocity $\omega_{N}$. The nucleus is assumed to be at the center of the structure and the rotation is in the plane defined by the loop centers. If the ring structure is designed so that $\omega_{r}$ matches $\omega_{N}$, it will be excited at the fundamental resonance. The radial component of this rotating magnetic field will induce a voltage $V_{n}$ in the $n$th loop. In general the relation between the induced voltages and the resulting currents is simply

$$
\underline{V}=\underline{\underline{Z}} \underline{\underline{I}},
$$

Here $\underline{V}$ and $\underline{I}$ are $N$-dimensional vectors, and $\underline{\underline{Z}}$ is an $N \times N$ matrix of the mutual impedances. Since the excitation is in the form of a wave traveling round the structure, the induced voltages must follow the same pattern. Hence the $n$th element of the vector $\underline{V}$ may be written as $V_{n}=V_{0}$ $\times \exp (-j 2 \pi n / N)$, where $V_{0}$ is a constant that can be determined from the geometry if the strength of the rotating magnetic dipole is known. Here $V_{0}$ will be taken as known and all the other quantities (current, output power) will be related to it.

In the nearest neighbor approximation the impedance matrix, denoted by $\underline{\underline{Z}}^{(0)}$ has the form

$$
\stackrel{\underline{Z}}{(0)}^{(0)}\left(\begin{array}{ccccccc}
Z_{0} & Z_{m} & 0 & 0 & \cdots & 0 & Z_{m} \\
Z_{m} & Z_{0} & Z_{m} & 0 & \cdots & 0 & 0 \\
0 & Z_{m} & Z_{0} & Z_{m} & \cdots & 0 & 0 \\
\cdots & \cdots & \cdots & \cdots & \cdots & \cdots & \cdots \\
0 & \cdots & \cdots & Z_{m} & Z_{0} & Z_{m} & 0 \\
0 & 0 & \cdots & 0 & Z_{m} & Z_{0} & Z_{m} \\
Z_{m} & 0 & \cdots & 0 & 0 & Z_{m} & Z_{0}
\end{array}\right) .
$$

The actual values of the mutual inductances for a particular geometry can be found by standard methods. ${ }^{33}$ Due to the circular symmetry of the excitation, the absolute values of all currents must be identical and the phases must follow those of the induced voltages. To see the relationship between the induced voltage and the current in a particular loop we can take (say) $n=2$, which, from Eq. (8), yields

$$
V_{2}=Z_{0} I_{2}+Z_{m}\left(I_{1}+I_{3}\right)=\left[Z_{0}+2 Z_{m} \cos (k d)\right] I_{2} .
$$

However, $k d$ is now impressed and is real and equal to $2 \pi / N$. Hence

$$
\operatorname{Im}\left[Z_{0}+2 Z_{m} \cos (2 \pi / N)\right]=0,
$$

whence we obtain

$$
I_{2}=V_{2} / R \text {. }
$$

This result (valid of course for all other elements) states that the current in element 2 under rotational resonance is the same as that obtained for a single uncoupled element excited by the same voltage at its resonant frequency, $\omega_{0}$. Such simple result can of course be obtained only at the frequency of rotational resonance. At other frequencies the current must be found numerically by inverting the voltage to current relationship, using

$$
\underline{I}=\left[\underline{\underline{Z}}^{(0)}\right]^{-1} \underline{V} .
$$

\section{B. Frequency variation of the current}

For an example numerical calculation, we assume that $\omega_{N} / 2 \pi=63.87 \mathrm{MHz}$, which would be obtained for ${ }^{1} \mathrm{H}$ NMR in a field strength of $1.5 \mathrm{~T}$. We also assume that $\omega_{r}=\omega_{N}$. For the loop parameters we choose $10 \mathrm{~mm}$ for the radius of the loop and $2 \mathrm{~mm}$ for the wire diameter. The value of the inductance calculated from the loop geometry ${ }^{34}$ is $L=33 \mathrm{nH}$ which leads then to a value of $187 \mathrm{pF}$ for the capacitance. The effect of losses (which limit the maximum power extractable) will be taken into account by considering three values of the quality factor $\left(Q_{0}=100,1000\right.$, and 10000) in all subsequent calculations. However, we accept that the latter two values can only, in practice, be realized by cooling the windings in the first case and by making them superconducting in the second. These $Q$ factors correspond to resistances of $0.132,0.0132$, and $0.00132 \Omega$.

The current flowing in an element (due to the circular symmetry all currents are equal) is of course frequency dependent. As the frequency departs from $\omega_{r}$ the current is bound to decline. The variation of the current follows a standard resonance curve, from which the quality factor $Q_{r}$ of the ring can be extracted. As may be expected, $Q_{r}$ is lower than $Q_{0}$. Numerical calculations yield $Q_{r}=94.3,944$, and 9420 for $Q_{0}=100,1000$, and 10000 .

\section{Extraction of power}

So far, the loops have been excited without extracting any power. We shall now compare the power that may be extracted from an element under rotational resonance with that obtained from a single uncoupled element at the same distance from the excitation. For the latter case the output power is

$$
P_{\text {out }, 0}=\left|V_{0}\right|^{2} Q_{0} / 8 \omega_{0} L, \quad\left|V_{0}\right|^{2}=\left(\mu_{0} H_{r} A\right)^{2},
$$

where $\mu_{0}$ is the free space permeability, $H_{r}$ is the radial component of the magnetic field at the center of the loop due to the rotating nuclear dipole, and $A$ is the area of the loop. For rotational resonance, we choose to extract power from the 
TABLE I. Optimum load reactance and resistance and the corresponding power ratio for $Q_{0}=100,1000$, and 10000

\begin{tabular}{rclc}
\hline \hline \multicolumn{1}{c}{$Q$} & $R_{L} / R$ & $X_{L} / R$ & $\zeta$ \\
\hline 100 & 4.18 & -2.73 & 6.03 \\
1000 & 11.5 & -0.076 & 11.8 \\
10000 & 12.0 & -0.0076 & 11.98 \\
\hline \hline
\end{tabular}

first element. The maximum power output, $P_{\text {out }, m}$, may be obtained by finding the optimum impedance $Z_{L}=R_{L}+j X_{L}$ to be inserted into this loop. The values of the optimum load impedance and those of the maximum power ratio

$$
\zeta=P_{\text {out }, m} / P_{\text {out }, 0}
$$

are shown in Table I. The results suggest that the limiting values of both the resistance ratio and the power ratio will approach $N / 2$ as $Q_{0}$ tends to infinity.

It is always desirable to check the numerical calculations by analytical methods whenever possible. We now do so, under conditions of rotational resonance and high $Q_{0}$. We first note that $\underline{\underline{Z}}^{(0)}$ in Eq. (8) is the unperturbed impedance matrix. We denote the perturbed matrix by $Z^{(L)}$; this matrix is identical with $\underline{\underline{Z}}^{(0)}$ except for the $\left[\underline{\underline{Z}}^{(0)}\right]_{11}$ element, which is changed from $Z_{0}$ to $Z_{0}+Z_{L}$. As a result, the first row of $\left[\underline{\underline{Z}}^{(L)}\right]^{-1}$ and $\left[\underline{\underline{Z}}^{(0)}\right]^{-1}$ are the same, but their determinants are different. Hence

$$
I_{1}^{(L)} / I_{1}^{(0)}=\left|\underline{\underline{Z}}^{(0)}\right| /\left|\underline{\underline{Z}}^{(L)}\right|,
$$

where $I_{1}^{(L)}$ and $I_{1}^{(0)}$ are the currents in the first loop under loaded and unloaded conditions at the frequency of the rotational resonance. In the Appendix, we show with the aid of Chebyshev polynomials that, in the limit when $Q_{0} \rightarrow \infty$, Eq. (15) may be expressed as

$$
\left|\underline{\underline{Z}}^{(0)}\right| /\left|\underline{\underline{Z}}^{(L)}\right|=\left[1+2 Z_{L} / N R\right]^{-1},
$$

whence the power ratio is

$$
(1 / 2)\left|I_{1}^{(L)}\right|^{2} R_{L} /(1 / 8)\left|I_{1}^{(0)}\right|^{2} R=\left(4 R_{L} / R\right)\left|1+2 Z_{L} / N R\right|^{-2} .
$$

Looking for the maximum of Eq. (17) we find the optimum values of the load reactance and resistance as $X_{L}=0$ and $R_{L}$ $=N R / 2$, and for the optimum power ratio we also obtain $\zeta$ $=N / 2$. Clearly the extrapolations made from the numerical results have been proved to be correct. It is also interesting to note [it follows from Eq. (15)] that the currents at rotational resonance obey again the same relationship as the currents in a simple resonant circuit - the current at optimum load is just one-half of that in the absence of a load.

In the unperturbed case, without power extraction, all the currents were shown to be of the same absolute value. Considering further the progressive variation of phase, the currents would constitute a circle in the complex plane. When the symmetry is disturbed, by extracting power from a particular element, the currents may no longer be expected to lie on a circle. Figures 3(a)-3(c) show the variation of the current with the element number in the complex plane at the rotational frequency, for $Q_{0}=100,1000$, and 10000 . Each plot is normalized so that the current in element 1 is real and the maximum value of the current is unity. For the lowest
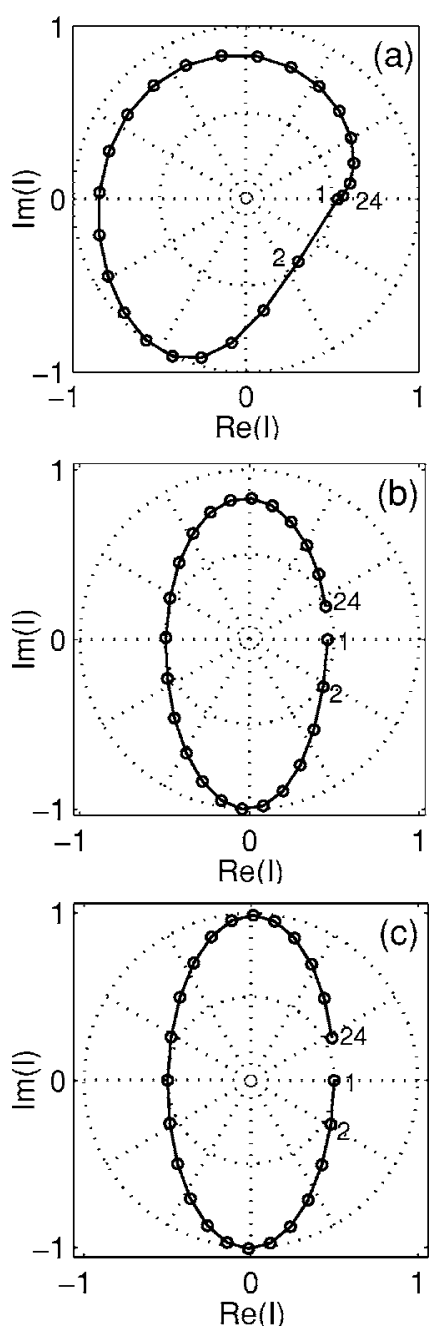

FIG. 3. Normalized currents in a 24 element ring with optimized load impedance plotted in the complex plane for (a) $Q_{0}=100$, (b) $Q_{0}=1000$, and (c) $Q_{0}=10000$. The numbering starts with element 1 , which contains the load, and increases clockwise.

value of $Q_{0}$ the curve is severely distorted. Near the element from which the power is extracted, the values of current are reduced. For $Q_{0}=1000$ some symmetry appears; the current is reduced where it is extracted and also on the opposite side. For $Q_{0}=10000$ the conclusions are the same, and the plot tends to an ellipse.

We wish to point out that the increase in output power when resonant elements are coupled to each other in a ring structure is analogous to what happens in a cavity magnetron. In that case the excitation is provided by electrons passing in front of the coupled resonant cavities, and the joint output is extracted from a single cavity.

At this stage the question arises whether calculations based on nearest neighbor interactions are sufficiently accurate. In order to give here an estimate of that accuracy we shall optimize the load impedance under conditions when all the interactions between the elements are taken into account. It leads to additional numerical calculations but the principle is simple. We need to calculate the mutual inductance between any two elements and insert its value into the $N \times N$ matrix shown in Eq. (8). The numerically optimized load impedance is found as $Z_{L} / R=10.4-3.12 j$ and $\zeta=11.36$. The 


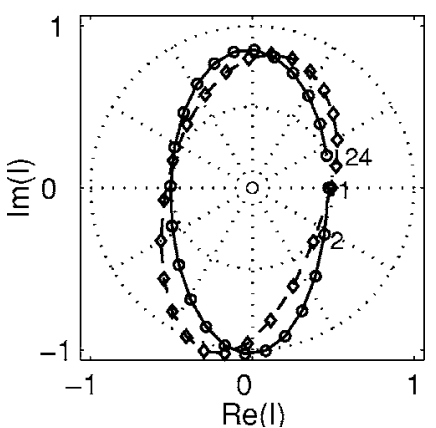

FIG. 4. Normalized current amplitudes in a 24 element ring with optimized load impedance plotted in the complex plane for $Q_{0}=1000$. Solid line: nearest neighbor interactions, dashed line: all interactions.

corresponding normalized current distribution (dashed line) is shown in Fig. 4 for $Q_{0}=1000$ together with the same relationship calculated from nearest neighbor interaction (solid line). The two curves look very similar. The main effect of taking all interactions into account is a consistent shift in the phases of the currents. When it comes to design there is no doubt that we shall have to take into account all possible interactions but for exploring the properties of the rotational resonance, as we do here, nearest neighbors lead to very good approximations. We shall therefore continue employing the simpler model in the rest of the paper.

\section{Increase of power output by additional matching impedances}

We have shown that under rotational resonance it is possible to obtain $N / 2$ times as much power out of a single loaded element as from an uncoupled loop. Is there room for further improvement? One feels intuitively that violating the circular symmetry, i.e., inserting a load in just one element, is a major perturbation that cannot possibly lead to an absolute maximum. One may argue then that its harmful effect can be mitigated (or even eliminated) by inserting further matching impedances. The right positions here are likely to be the nearest neighbors. We shall therefore insert reactances (denoted by $X_{N, a}$ and $\left.X_{2, a}\right)$ into elements $N$ and 2. However, in the present case the optimum load impedance will also depend on the matching reactances. Hence we need to optimize simultaneously $Z_{L}, X_{N, a}$, and $X_{2, a}$. Their values and those of $\zeta$ can be found in Table II. For the power ratio the trend is clear: $\zeta$ tends to $N$ as $Q_{0}$ increases. We hope to provide an analytical proof in a future paper. We have calculated the corresponding normalized current distributions and found them to lie closer to the circle of unit radius than those in Fig. 3, as expected. In fact, for $Q_{0}=10000$ the current distribution practically coincides with the unit circle.

TABLE II. Optimum load reactance, resistance, matching impedances, and the corresponding power ratio for $Q_{0}=100,1000$, and 10000

\begin{tabular}{rccccc}
\hline \hline \multicolumn{1}{c}{$Q$} & $R_{L} / R$ & $X_{L} / R$ & $X_{N, a} / R$ & $X_{2, a} / R$ & $\zeta$ \\
\hline 100 & 5.96 & -2.87 & 8.74 & -3.46 & 8.49 \\
1000 & 21.1 & -3.23 & 32.02 & -16.2 & 23.5 \\
10000 & 22.68 & -0.45 & 23.51 & -21.85 & 23.96 \\
\hline \hline
\end{tabular}

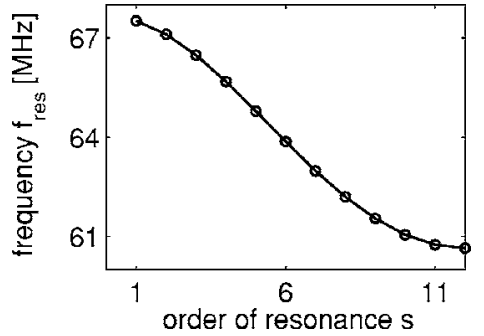

FIG. 5. Frequenćies of the higher order resonances.

\section{E. Higher order resonances}

A higher order rotational resonance occurs when, as mentioned in Sec. II, the perimeter of the resonant structure is equal to $s \lambda$. Smaller MI wavelength means higher value of $\beta d$ and correspondingly lower value of frequency. The highest distinguishable resonance occurs for $s=N / 2$, when the amplitude of the wave changes sign from element to element. The variation of the rotational resonant frequency with the order of the resonance is shown in Fig. 5 for $N=24$. This relationship is somewhat at variance with expectations. The frequency of higher order resonances in familiar electromagnetic or acoustic resonators rises with the order. For MI waves in the present planar configuration, higher order resonances occur at lower frequencies because the wave is a backward one.

The current distribution for higher order resonances may be determined by the same technique as before, by numerically optimizing the load impedance in element 1 and the reactances in the neighboring elements (their values are shown in the caption of Fig. 6). The main difference is that one needs an excitation that varies correspondingly to excite a higher resonance. Here, we consider the case of $s=2$, which requires a quadrupole excitation, i.e., a voltage varying as $V_{n}=V_{0} \exp (-j 4 \pi n / N)$. The resultant current distribution is plotted in the complex plane in Fig. 6, with all the other parameters as before. The period has clearly been halved.

\section{F. The inverse problem}

We have shown that a traveling wave voltage excitation leads to a current distribution which, when terminated by an optimum load, becomes more and more akin to a traveling

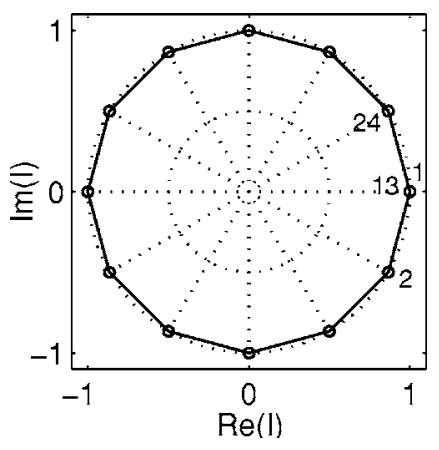

FIG. 6. Normalized currents for the second rotational resonance in a 24 element ring plotted in the complex plane. $Z_{L}=0.0294-0.0004 j \Omega, X_{N, a}$ $=0.0226 \Omega$, and $X_{2, a}=-0.0209 \Omega$. 

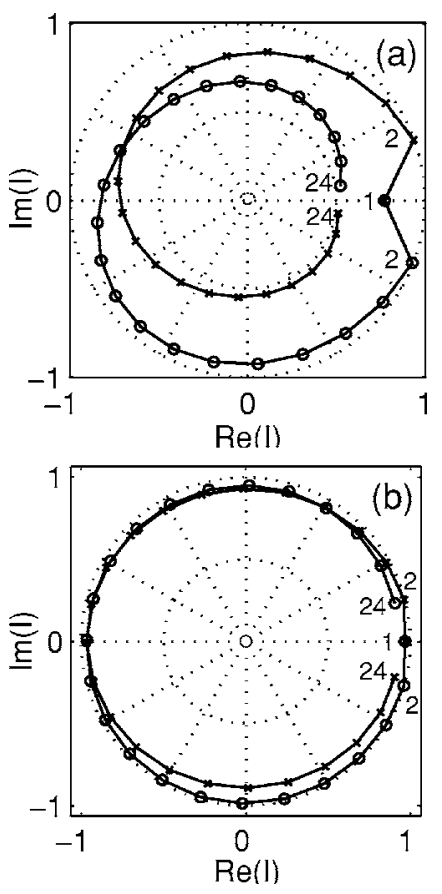

FIG. 7. Normalized currents in a 24 element ring excited by a rotating dipole (circles) and by a voltage applied to the load (crosses) for (a) $Q_{0}$ $=1000$ and $(\mathrm{b}) Q_{0}=10000$.

wave distribution as $Q_{0}$ increases. Is the converse true? Having determined the optimum load and matching impedances, can we then omit the exciting magnetic dipole and obtain a traveling wave current in the loops by applying a voltage to the optimized load? We have chosen for illustration two cases with optimized load and matching reactances using the normalization procedure of Fig. 3. The corresponding current distributions are shown in Figs. 7(a) and 7(b) for $Q_{0}=1000$ and 10000 , respectively. Note that the rotating magnetic dipole gives rise to a clockwise varying phase distribution (denoted by circles). In contrast, a voltage applied to the load induces a current distribution (denoted by crosses) with an anticlockwise phase variation. The two counterpropagating current distributions have similar, but not identical, amplitudes. For $Q_{0}=10000$ the two distributions are very close to circles suggesting that in the absence of losses the direct and inverse problems have the same current distributions, and their only difference is the direction of travel.

\section{G. Dependence on the various parameters}

In order to perform the various numerical calculations we had to choose actual values for a number of parameters such as the radius of the structure, the number of elements, the value of the loop inductance, and the frequency of operation. Obviously there is a need to see what happens as all these parameters are varied. While investigating many examples, we have found that changing the parameters introduces only minor variation, leaving the conclusions unchanged. We have shown that with the aid of rotational resonance up to $N$ times more power can be extracted from a single matched coil. However, two important questions remain unanswered: what is the optimum shape of the resonant coil and what is the optimum number of elements? It is un- likely that the circular shape (adopted here for simplicity) would be optimum. A rectangular form would be more efficient picking up power from the rotating dipole and it might even be desirable to fit the coil over a spherical surface. The optimum number of elements would depend on the size and shape of the sample and would require a definition of optimum performance (e.g., to maximize the signal to noise ratio) which we have been unable to discuss in the present paper but hope to return to it in a future publication.

\section{APPLICATIONS TO NMR AND FUTURE WORK}

MRI involves signal acquisition from very large numbers of sources (known as voxels) distributed throughout a volume that potentially occupies much of the interior of any detector. The analysis in this paper does not describe this situation. On the other hand, MRS in vivo is frequently performed on single voxels which, because of the low concentrations of many of the metabolites of interest, are generally much larger than those used in MRI. Techniques such as image-selected in vivo spectroscopy ${ }^{35}$ (ISIS) (for phosphorus) and stimulated echo acquisition mode ${ }^{36}$ (STEAM) (for protons) use single voxels located centrally in the tissue. The performance of MRS is limited by the signal to noise ratio, and the signal gain described here may offer a potential solution. The parameters we have assumed (for example, the ring radius) are appropriate to the study of the pediatric brain, and we hope to investigate this application in the future.

The present work can be extended in other directions. For example, the planar configuration could be replaced by a quasiaxial one, allowing the elements to be placed closer together. It would also be desirable to investigate the cases when the nuclei exciting the MI wave are offset from the center of the ring, as occurs in most NMR studies, or when the number of elements to be detected is much larger and they are widely distributed (as in MRI). Another interesting line of study would be to investigate the response when a nonlinear element (a varactor) is inserted in the loop, when it should be possible to achieve parametric amplification of the signal at the source. Another set of problems to be studied is the use of the phenomenon of rotational resonance for determining the position of a small number of rf sources, which are used to track inserted devices.

\section{CONCLUSIONS}

The propagation of MI waves on a circular set of resonant loops has been studied when excited by a rotating magnetic dipole located at the center of the structure. The analytical calculations have been based on nearest neighbor interactions which were shown to give good approximation by comparing it with a numerical approach that included all interactions between the elements. It has been shown that such waves can have resonances (called rotational resonances) when the perimeter is equal to an integral number of MI wavelengths. Optimum conditions for extracting power from a loaded loop in a chain of $N$ loops have been determined. It has been proven analytically and numerically that the extractable power is $N / 2$ (without additional matching 
elements) or $N$ times (with such elements) larger than that obtainable from a single uncoupled resonant loop. The inverse problem of applying a voltage to a single element containing the optimized load has also been studied and it has been shown that traveling wave currents can be obtained. Applications in MRS have been identified.

\section{ACKNOWLEDGMENTS}

Three of the authors (O.Z., O.S. and E.S.) acknowledge financial support by the German Research Council (Emmy Noether-Program and Graduiertenkolleg 695).

\section{APPENDIX}

We shall find here an analytic expression for $\left|\underline{\underline{Z}}^{(0)}\right| /\left|\underline{\underline{Z}}^{(L)}\right|$ in Eq. (15) when the quality factor tends to infinity. We start with a normalization procedure dividing each element of the matrix in Eq. (8) by $Z_{m}$ and introducing the notation

$$
x=Z_{0} / 2 Z_{m}=-\cos (k d) .
$$

The resulting $N \times N$ matrix will be denoted by $\underline{\underline{\delta}}^{(0)}$ and will take the form

$$
\underline{\delta}^{(0)}=\left(\begin{array}{ccccccc}
2 x & 1 & 0 & 0 & \cdots & 0 & 1 \\
1 & 2 x & 1 & 0 & \cdots & 0 & 0 \\
0 & 1 & 2 x & 1 & \cdots & \cdots & \cdots \\
\cdots & \cdots & \cdots & \cdots & \cdots & \cdots & \cdots \\
\cdots & \cdots & \cdots & \cdots & \cdots & \cdots & \cdots \\
0 & \cdots & \cdots & \cdots & 1 & 2 x & 1 \\
1 & 0 & \cdots & \cdots & 0 & 1 & 2 x
\end{array}\right) .
$$

For the derivation to follow we shall need to define three further matrices.

(1) $\underline{\underline{\delta}}^{(L)}$, which is identical with $\underline{\underline{\delta}}^{(0)}$ apart from the $(1,1)$ element which is $2 x+Z_{L} / Z_{m}$.

(2) $\underline{\underline{U}}$, which is identical with $\underline{\underline{\delta}}^{(0)}$ apart from the $(1, N)$ and $(N, 1)$ elements which are equal to zero (physically this is the normalized impedance matrix of a linear array, in which the first and $N$ th elements are not coupled to each other)

(3) $T$ is identical with $\underline{\underline{U}}$ apart from the $(1,1)$ element, which is equal to $x$ instead of $2 x$.

The determinants of these $N \times N$ matrices will be denoted by $\delta_{N}^{(0)}, \delta_{N}^{(L)}, U_{N}$, and $T_{N}$. The aim is to express

$$
\left|\underline{\underline{Z}}^{(0)}\right| /\left|\underline{\underline{Z}}^{(L)}\right|=\delta_{N}^{(0)} / \delta_{N}^{(L)}
$$

with the aid of $T_{N}$ and $U_{N}$ which define, respectively, the first and second kind of the $N$ th order Chebyshev polynomial. ${ }^{37}$ We also need the alternative definitions

$$
T_{N}[\cos (q)]=\cos (N q)
$$

and

$$
U_{N}[\cos (q)]=\sin [(N+1) q] / \sin (q) .
$$

Next we can show that

$$
\delta_{N}^{(L)}=\delta_{N}^{(0)}+\left(Z_{L} / Z_{m}\right) U_{N-1},
$$

with which Eq. (A1) modifies to

$$
\left|\underline{\underline{Z}}^{(0)}\right| /\left|\underline{\underline{Z}}^{(L)}\right|=\left[1+\left(Z_{L} / Z_{m}\right)\left(U_{N-1} / \delta_{N}^{(0)}\right)\right]^{-1} .
$$

It may be shown from simple determinant relationships that

$$
\delta_{N}^{(0)}=2\left[T_{N}-(-1)^{N}\right] .
$$

Using now Eq. (A1) and the definition of Eq. (A4), and performing a number of trigonometric operations, Eq. (A6) reduces to

$$
U_{N-1} / \delta_{N}^{(0)}=-\sin (N k d)\{2 \sin (k d)[\cos (N k d)-1]\}^{-1} .
$$

Under conditions of rotational resonance

$$
k d=2 \pi / N-j \alpha d .
$$

Assuming further that losses are very small (i.e., $Q_{0} \rightarrow \infty$ ) and using Eq. (5) we find the required result

$$
U_{N-1} / \delta_{N}^{(0)}=2 Z_{m} / N R
$$

whence Eq. (16) follows.

${ }^{1}$ V. G. Veselago, Sov. Phys. Usp. 10, 509 (1968).

${ }^{2}$ W. N. Hardy and L. A. Whitehead, Rev. Sci. Instrum. 52, 213 (1981).

${ }^{3}$ H. J. Schneider and P. Dullenkopf, Rev. Sci. Instrum. 48, 68 (1977).

${ }^{4}$ B. T. Ghim, G. A. Rinard, W. Quine, S. S. Eaton, and G. R. Eaton, J. Magn. Reson., Ser. A 120, 72 (1996).

${ }^{5}$ W. Rotman, IRE Trans. Antennas Propag. 10, 82 (1962).

${ }^{6}$ J. B. Pendry, A. S. Holden, D. J. Robbins, and W. J. Stewart, IEEE Trans. Microwave Theory Tech. 47, 2075 (1999).

${ }^{7}$ D. R. Smith, W. J. Padilla, D. C. Vier, S. C. Nemat-Nasser, and S. Schultz, Phys. Rev. Lett. 76, 2480 (2000).

${ }^{8}$ R. A. Shelby, D. R. Smith, and S. Schultz, Science 292, 77 (6 April 2001).

${ }^{9}$ R. G. E. Hutter, Beam and Wave Electronics in Microwave Tubes (Van Nostrand, Princeton, 1960).

${ }^{10}$ I. V. Lindell, S. A. Tretyakov, K. I. Nikoskinen, and S. Ilvonen, Microwave Opt. Technol. Lett. 31, 129 (2001).

${ }^{11}$ M. Gorkunov, M. Lapine, E. Shamonina, and K. H. Ringhofer, Eur. Phys. J. B 28, 263 (2002)

${ }^{12}$ M. Quinton, A. Leitner, J. R. Krenn, and F. R. Aussenegg, Opt. Lett. 23, 1331 (1998).

${ }^{13}$ M. L. Brongersma, J. W. Hartman, and H. A. Atwater, Phys. Rev. B 62, R16356 (2000).

${ }^{14}$ W. H. Weber and G. W. Ford, Phys. Rev. B 70, 125429 (2004).

${ }^{15}$ S. A. Tretyakov and A. J. Viitanen, Electr. Eng. 82, 353 (2000).

${ }^{16}$ E. Shamonina, V. A. Kalinin, K. H. Ringhofer, and L. Solymar, Electron. Lett. 38, 371 (2002).

${ }^{17}$ L. Brillouin, Wave Propagation in Periodic Structures (McGraw-Hill, London, 1946).

${ }^{18}$ E. Shamonina, V. A. Kalinin, K. H. Ringhofer, and L. Solymar, J. Appl. Phys. 92, 6252 (2002).

${ }^{19}$ M. C. K. Wiltshire, E. Shamonina, I. R. Young, and L. Solymar, Electron. Lett. 39, 215 (2003)

${ }^{20}$ M. C. K. Wiltshire, E. Shamonina, I. R. Young, and L. Solymar, J. Appl. Phys. 95, 4488 (2004).

${ }^{21}$ M. J. Freire, R. Marques, F. Medina, M. A. G. Laso, and F. Martin, Appl. Phys. Lett. 85, 4439 (2004).

${ }^{22}$ I. S. Nefedov and S. A. Tretyakov, Microwave Opt. Technol. Lett. 45, 98 (2005).

${ }^{23}$ M. J. Freire and R. Marques, Appl. Phys. Lett. 86, 182505 (2005).

${ }^{24}$ M. C. K. Wiltshire, J. B. Pendry, I. R. Young, D. J. Larkman, D. J. Gilderdale, and J. V. Hajnal, Science 291, 849 (2001).

${ }^{25}$ P. B. Roemer, W. A. Edelstein, and C. E. Hayes, in Methods in Biomedical Magnetic Resonance Imaging and Spectroscopy, edited by I. R. Young (Wiley, Chichester, 2000), p. 151.

${ }^{26}$ C. E. Hayes, Birdcage Resonators: Highly Homogeneous Radiofrequency Coils for Magnetic Resonance Imaging and Spectroscopy (Wiley, Chichester,2000), p. 136

${ }^{27}$ K. L. Meyer and D. Ballon, J. Magn. Reson., Ser. B 107, 19 (1995). 
${ }^{28}$ R. A. Silin and V. P. Sazonov, Slow Wave Structures (Boston SPA, Eng. National Lending Library for Science and Technology, 1971).

${ }^{29}$ G. I. Atabekov, Linear Network Theory (Pergamon, Oxford, 1965).

${ }^{30}$ R. R. A. Syms, E. Shamonina, V. Kalinin, and L. Solymar, J. Appl. Phys 97, 064909 (2005).

${ }^{31} \mathrm{~K}$. Chang, Microwave Ring Circuits and Antennas (Wiley, New York, 1996).

${ }^{32}$ J. T. Randall, Proc. Phys. Soc. London 58, 247 (1946).

${ }^{33}$ L. Solymar, Electromagnetic Theory, 2nd ed. (Oxford University Press, Oxford, 1981).
${ }^{34}$ F. W. Grover, Inductance Calculations (Dover, New York, 1946).

${ }^{35}$ R. J. Ordidge, A. Connelly, and J. A. B. Lohman, J. Magn. Reson. (19691992) 66, 283 (1986).

${ }^{36}$ J. Frahm, M. L. Gyngell, H. Bruhn, K. D. Merboldt, W. Hanicke, and R. Sauter, Proceedings of the Seventh Annual Meeting of Society of Magnetic Resonance in Medicine, San Francisco (ISMRM, Berkeley, CA, 1988), p. 613 .

${ }^{37}$ T. J. Rivlin, Chebyshev Polynomials: from Approximation Theory to Algebra and Number Theory, 2nd ed. (Wiley, New York, 1990). 